Provided for non-commercial research and education use. Not for reproduction, distribution or commercial use.

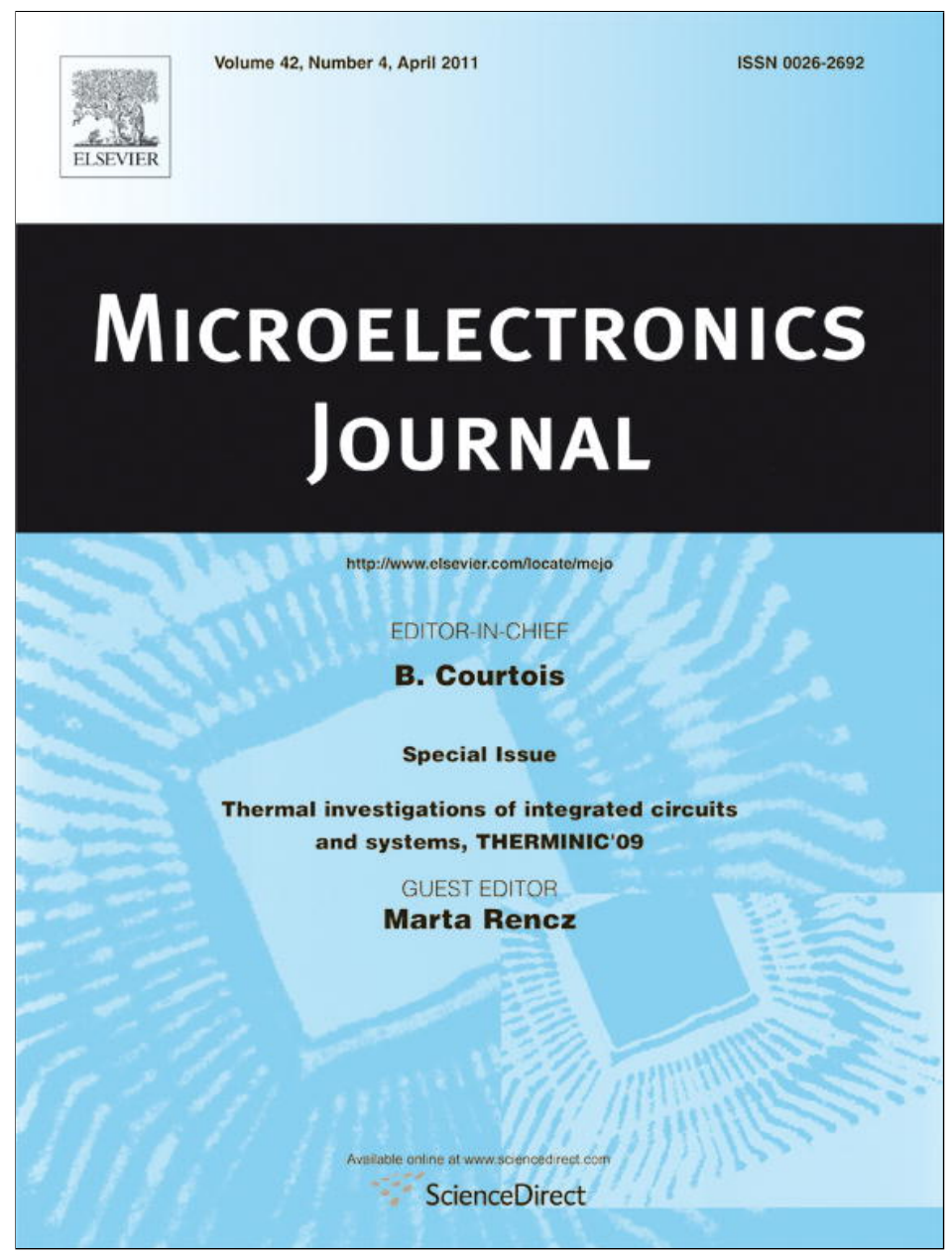

This article appeared in a journal published by Elsevier. The attached copy is furnished to the author for internal non-commercial research and education use, including for instruction at the authors institution and sharing with colleagues.

Other uses, including reproduction and distribution, or selling or licensing copies, or posting to personal, institutional or third party websites are prohibited.

In most cases authors are permitted to post their version of the article (e.g. in Word or Tex form) to their personal website or institutional repository. Authors requiring further information regarding Elsevier's archiving and manuscript policies are encouraged to visit:

http://www.elsevier.com/copyright 


\title{
Emulation-based transient thermal modeling of 2D/3D systems-on-chip with active cooling
}

\author{
Pablo G. Del Valle ${ }^{\mathrm{a}, \mathrm{b}, *}$, David Atienza ${ }^{\mathrm{b}}$ \\ a Departamento de Arquitectura de Computadores y Automática (DACYA), UCM, Avda. Complutense s/n, 28040 Madrid, Spain \\ ${ }^{\mathrm{b}}$ Embedded Systems Laboratory (ESL), EPFL, Station 11, EPFL-STI-IEL-ESL, CH-1015 Lausanne, Switzerland
}

\section{A R T I C L E I N F O}

\section{Article history:}

Received 3 December 2009

Received in revised form

28 July 2010

Accepted 6 August 2010

Available online 21 September 2010

Keywords:

Thermal modeling

Transient temperature analysis

FPGA emulation

2D/3D MPSoC

Active cooling

Close-loop systems

\begin{abstract}
A B S T R A C T
State-of-the-art devices in the consumer electronics market are relying more and more on MultiProcessor Systems-On-Chip (MPSoCs) as an efficient solution to meet their multiple design constrains, such as low cost, low power consumption, high performance and short time-to-market. In fact, as technology scales down, logic density and power density increase, generating hot spots that seriously affect the MPSoC performance and can physically damage the final system behavior. Moreover, forthcoming three-dimensional (3D) MPSoCs can achieve higher system integration density, but the aforementioned thermal problems are seriously aggravated. Thus, new thermal exploration tools are needed to study the temperature variation effects inside 3D MPSoCs. In this paper, we present a novel approach for fast transient thermal modeling and analysis of 3D MPSoCs with active (liquid) cooling solutions, while capturing the hardware-software interaction. In order to preserve both accuracy and speed, we propose a close-loop framework that combines the use of Field Programmable Gate Arrays (FPGAs) to emulate the hardware components of 2D/3D MPSoC platforms with a highly optimized thermal simulator, which uses an RC-based linear thermal model to analyze the liquid flow. The proposed framework offers speed-ups of more than three orders of magnitude when compared to cycleaccurate 3D MPSoC thermal simulators. Thus, this approach enables MPSoC designers to validate different hardware- and software-based 3D thermal management policies in real-time, and while running real-life applications, including liquid cooling injection control.
\end{abstract}

(c) 2010 Elsevier Ltd. All rights reserved.

\section{Introduction}

The power density of high performance systems continues to increase with every process technology generation. Nowadays, several commercial multi-processor system-on-chip (MPSoC) architectures are available with several tens of cores, such as IBM's Cell [1], Sun's Niagara T1 [2] and Tilera's 64-core architecture [3]. However, in these new MPSoC architectures, power density increases the operating temperature and creates significant hot-spots on the die that need to be managed. Furthermore, 3D stacking is an emerging solution to increase the integration capabilities and frequency of forthcoming MPSoCs [4,5], but it substantially increases further power density due to the placement of computational units on top of each other. Therefore, temperature-induced problems exacerbate in 3D systems and are a major concern to be explored as early as possible in 3D MPSoC design and integration.

* Corresponding author at: Departamento de Arquitectura de Computadores y Automática (DACYA), UCM, Avda. Complutense s/n, 28040 Madrid, Spain. Tel.: + 34913947541.

E-mail address: pablo.garciadelvalle@epfl.ch (P.G. Del Valle).
To explore the hardware/software (HW/SW) thermal interaction, cycle-accurate MPSoC simulators including SW thermal models exist, based on post-processing of run-time power consumption and floorplanning information [6-8]. However, these complex SW environments are very limited in performance (i.e., up to $100 \mathrm{kHz}$ ) due to signal management overhead and are not interactive with thermal control systems in real-time. Thus, they are not suitable for thermal control exploration in 2D/3D MPSoCs running complex real-life applications. Moreover, higher abstraction levels simulators attain faster simulation speeds, but lose significantly the accuracy for fine-grained thermal-aware architectural tuning or thermal modeling.

One alternative to cycle-accurate simulators is HW emulation. Various MPSoC emulation frameworks have been proposed [9-11]. Nevertheless, they are not designed for thermal exploration and are usually very expensive (between $\$ 100 \mathrm{~K}$ and $\$ 1 \mathrm{M}$ ) and not flexible enough for MPSoC architecture exploration since their baseline architectures (e.g., processing cores or interconnections) are proprietary, not permitting internal changes. Furthermore, no flexible interconnection interfaces between HW emulation and also no fast thermal libraries that model active cooling behavior (e.g., liquid cooling $[12,13]$ ) exist nowadays. Thus, thermal effects can only be 
verified in the last phases of the design process, typically when the final architecture and cooling components are available can be tested in the final system integration process, which can typically result in very expensive redesigns.

As a result, one major design challenge is the deployment of fast exploration methods of multiple HW and SW implementation alternatives for 2D and 3D MPSoCs with accurate estimations (e.g., performance, energy) that address the modeling of transient thermal behavior to tune the final architectures.

In this paper, we present a new HW/SW FPGA-based emulation framework of the 2D/3D MPSoC architectures, which enables realistic thermal studies at an early stage of system integration, including active (single-phase liquid) cooling modeling, as well as power, energy and performance constraints validation in realtime. First, the HW components of the system are mapped on an FPGA and statistics are extracted from three key MPSoC architectural levels (processors, memory subsystem and interconnections), while real-life applications are executed. Second, this run-time information is sent using a standard Ethernet connection to a dynamically adaptable SW thermal modeling tool running on a host PC. Third, this tool evaluates in real-time the thermal behavior of the final MPSoC design, selecting different ordinary differential equation solvers according to the desired accuracy in thermal exploration and simulation time of 2D/3D chip stacks, and returns this information to the FPGA emulating the design. This final step creates a closed-loop thermal simulation environment for $2 \mathrm{D}$ and $3 \mathrm{D}$ chips that enables testing temperature management strategies in real-time.

The experimental results with 2D/3D MPSoCs, using real-life case studies models of the UltraSPARC T1 [2] and other industrial platforms from Freescale [14], Philips [15], etc., show that this HW/SW emulation framework for transient thermal analysis can achieve speed-ups of more than three orders of magnitude compared to state-of-the-art cycle-accurate thermal simulators, while keeping the accuracy of uncertainty levels of the simulated temperature obtained with the proposed method within $3 \%$ with respect to finite-element simulations.

The remainder of this paper is structured as follows: It starts in Section 2 with a detailed overview of prior art in thermal modeling and architectural simulation for 2D and 3D MPSoCs. Then, in Section 3 the proposed HW/SW thermal emulation flow is presented. Next, in Section 4 the 3D liquid cooling model is described. After that, in Section 5 we present the experimental setup and results, performed with different 2D and 3D MPSoCs to validate the simulation model and to demonstrate the ease of use of the platform. Finally, in Section 6 we summarize the main conclusions of this work.

\section{Related work}

It is widely accepted that $2 \mathrm{D} / 3 \mathrm{D}$ MPSoCs represent a promising solution for forthcoming complex processing systems [18]. This has spurred research on modeling and prototyping MPSoC designs, using both HW and SW. From the SW viewpoint, solutions have been suggested at different abstraction levels, enabling tradeoffs between simulation speed and accuracy. First, fast analytical models have been proposed to prune very distinct design options using high-level languages (e.g., C or C+) [19]. Also, full system simulators, like Symics [20] and others [7,8], have been developed for embedded SW debugging and can reach megahertz speeds, but are not able to accurately capture performance and power effects (e.g., at the interconnection level) depending on the cycle-accurate behavior of the HW. Second, transaction-level modeling in SystemC, in academic [21] and industrial context $[22,23]$ has enabled more accuracy in system-level simulation at the cost of sacrificing simulation speed (about 100-200 kHz). Such speeds render unfeasible the transient testing of large systems due to overly long simulation times, conversely to the proposed 2D/3D thermal emulation framework. Moreover, in most cases SW simulations are only limited to a number of proprietary interfaces.

Finally, important research has been done to obtain cycleaccurate frameworks in low-level SystemC or hardware description languages (HDL). Companies and universities have developed cycle-accurate simulators using post-synthesis libraries from HW vendors [27,28]. However, their simulation speeds $(10-120 \mathrm{kHz})$ are unsuitable for long MPSoC thermal exploration.

The most important alternative nowadays to simulation is HW emulation. In industry, one of the most complete sets of statistics is provided by Palladium II [9], which can accommodate very complex systems (i.e., up to 256 Mgate). However, its main disadvantages are its operation frequency (approximately $1.6 \mathrm{MHz}$ ) and cost (around $\$ 1$ million). Then, ASIC integrator [10] is much faster for architectural exploration. Nevertheless, its major drawback is its limitation to only up to few ARM-based cores and only AMBA interconnects. The same exploration limitation of proprietary cores occurs with Heron SoC emulation [24] and Zebu-XL [11], both based on multi-FPGA emulation in the order of $\mathrm{MHz}$. They can be used to validate intellectual property blocks, but are not flexible enough for fast MPSoC design exploration or detailed statistics extraction. In the academic world, a recent emulation platform for exploring performance of MPSoC alternatives is TC4SOC [25]. It uses a proprietary 32-bit VLIW core and enables exploration of interconnects by using an FPGA, but, it does not enable detailed extraction of statistics and performing thermal modeling at the other three architectural levels proposed in this work, i.e., memory hierarchy, interconnects and processing cores. Finally, an interesting approach that uses FPGA prototyping to speed-up co-verification of pure SW simulators is described in [26], which uses a cycle-by-cycle synchronization basis with the $\mathrm{C} / \mathrm{C}+\mathrm{SW}$ part by using an array of shared registers in the FPGA that can be accessed by both sides at a speed of $1 \mathrm{MHz}$, outlining the potential benefits of combined HW-SW frameworks which is exploited in the proposed approach to reach an emulation speed of hundreds of MHzs.

Turning our attention to thermal modeling, [6] presented a thermal/power model for 2D super-scalar architectures. It can predict the temperature variations between the different components of a processor and show the expected influence in performance. Additionally, $[14,17]$ have investigated the impact of temperature and voltage variation across the die of 2D and 3D MPSoCs. Their results show that the temperature can vary by more than $25^{\circ}$ across the die and tiers. In all these works, a "1D" approximation is often assumed to evaluate the thermal behavior $[29,30]$. This means that the power is uniformly produced on active levels (or on parts of them), one per stratum. This assumption may lead to strongly underestimated maximum temperature. Thus, several authors [31] use this simplification but perform detailed simulation of 3D thermal effects due to the presence and localization of supervias, and analyze the local (3D) and global (1D) modeling contribution to the maximum temperature, showing that thermal resistance can be higher than 1D thermal resistance due to local 3D effects and even more finegrain transient analysis need to be performed to avoid thermal overestimations.

Finally, numerical thermal simulations have been carried out to convert power dissipation distribution into a temperature distribution in a three-dimensional integrated circuit (3D-IC) [32]. Based on the past work, the development of a fundamental analytical model for heat transport in 3D integrated circuits is highly desirable. Such an analytical model provides a framework 
for the analysis of the general problem of heat dissipation in 3D ICs, and will enable simple thermal design guidelines.

A key component of the 3D technology is the through-silicon via (TSV) that enables communication between the two dies as well as with the package. Several works have analyzed the optimization of placement of TSVs for heat dissipation in 3D ICs [31,32]. Other works [33] propose analytical and finite-element models of heat transfer in 3D electronic circuits and use this model to analyze the impact of various geometric parameters and thermo-physical properties (through silicon vias, inter-die bonding layers, etc.) on thermal performance, but at the cost of very time-consuming simulations.

Overall, all of these works prove the importance of hot spots in 2D high-performance multi-core systems (and even more in 3D structures), as well as the need of accurate and fast transient temperature analysis tools for the different architectural components of MPSoCs (cores, TSVs, etc.). Thus, the proposed emulation method aims at estimating accurately the transient temperature of integrated circuits implementing 2D/3D MPSoCs, including active cooling mechanisms (e.g., liquid cooling).

\section{HW/SW closed-loop thermal emulation flow for 2D/3D MPSoCs}

\subsection{Closed-loop emulation flow}

Fig. 1 depicts an overview of the instantiation of the proposed HW/SW thermal emulation environment for a Freescale-based 3-core MPSoC [14], implemented onto a Xilinx Virtex-V FPGA, modeling the transient thermal behavior of the system while executing multiple multimedia applications (e.g., SW-defined radio, video streaming, etc.) and a multi-processor operating system (OS). The system can be scaled to any number of cores subsystems by using appropriate FPGAs.

The instantiation flow of the proposed HW/SW transient thermal emulation environment is created in four steps:

1. The HW part of the emulator is defined. It implies synthesizing the MPSoC architecture onto a certain FPGA target technology (a multi-FPGA environment exists, if it is necessary).

2. Specific hardware sniffers (transparent to the normal MPSoC operation) are included in the FPGA that monitor particular signals of each component of the target 2D/3D architecture [15].

3. The run-time power information (frequency/voltage and leakage power) of each system component is sent to a SW thermal modeling tool running on a host PC that evaluates in real-time

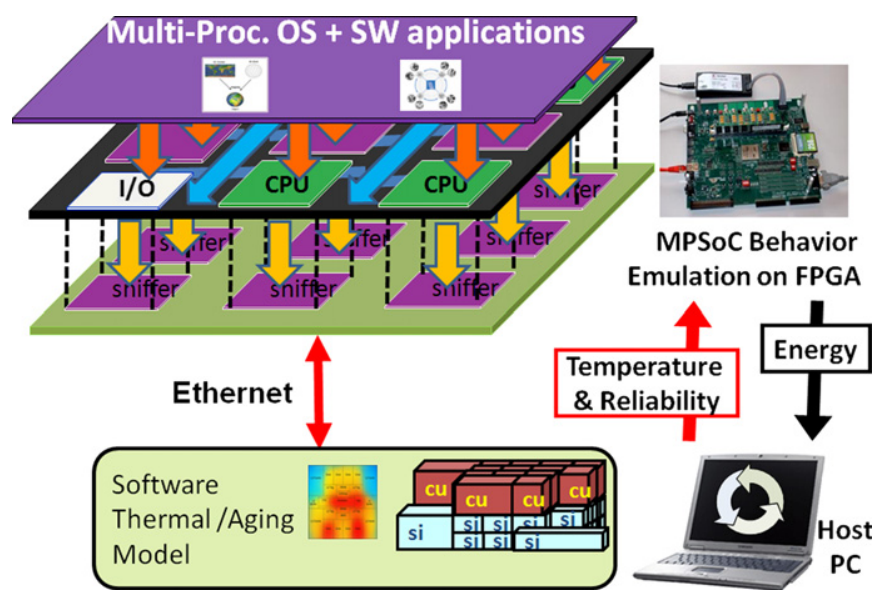

Fig. 1. Overview of the 2D/3D MPSoC thermal emulation framework. the thermal behavior of the final 2D/3D MPSoC design using a thermal model developed for bulk silicon chip systems [15], and calculates the temperature of each cell in the floorplan of the emulated system. The model includes different types of ordinary differential equation solvers [16] (Forward Euler 1st order, Crank-Nicholson 2nd order method, Runge-Kutta 4th order method, etc.), which enables multiple trade-offs between accuracy and thermal modeling time in 2D/3D chip stacks.

4. The temperatures calculated by the SW thermal library are sent back to the FPGA emulating the MPSoC system (see link between the host PC and the FPGA on the right side of Fig. 1) and are stored in registers of the FPGA that emulate the presence of thermal sensors in the target MPSoC in certain positions of the floorplan. The registers containing the predicted temperature are mapped in the memory hierarchy, so that they can be accessed from the running multi-processor OS, providing real-time temperature information, and making up a closed-loop thermal monitoring system

The closed loop scheme, mentioned in step four, is really needed. First obvious advantage is that the system becomes aware of its own temperature (through the reading of the integrated thermal sensors), thus, the on-board OS can make decisions at runtime based on this information, e.g., reducing the frequency/voltage of some of the cores, migrating tasks, shutting down processors, etc. The second, and not so straightforward, implication of the closed loop is the effects the temperature has on the thermal behavior of MPSoCs. It would be impossible to perform an accurate estimation of the temperature without this knowledge. The thermal model needs to be constantly adjusted as the temperature varies, since temperature affects the leakage, and leakage directly affects power consumption of the chip.

\subsection{Modeling a 3D chip with an FPGA}

To understand how we model a 3D architecture using a 2D FPGA, take a look at the 3D system depicted in Fig. 1a. It consists of two layers: In the upper layer there is a core that can access two local memories: "A" (in the same layer), and "B" (located in the lower layer). An access to memory A will take less time and will consume less power than accessing $\mathrm{B}$.

When emulating this system in an FPGA, we have to map everything in a 2D layout. If we abstract the floorplanning information, what is different in the behavior of systems "a" and " $b$ " is the latency. Assume, for example, that accessing memory A takes 1 cycle, whereas accessing memory B takes 6 cycles. We instantiate in the FPGA a processor connected to two memories symmetrically and, then, we simply add a new element that will simulate this extra latency (red oval in the figure). The behavior of the system will be the same as the one in the 3D case.

From the point of view of the thermal model, the data interface remains the same: we only receive "activity numbers" associated to the different elements of the floorplan (number of accesses to memory $\mathrm{X}$, number of transactions in bus $\mathrm{Y}$, etc.), so there is no difference.

Nevertheless, when calculating temperatures, the thermal model knows that the bus of memory A is different from the bus of memory B: different materials, capacitances, etc. It should be noted that inside the FPGA it is completely irrelevant where we place memory $\mathrm{B}$, as far as the behavior is the same (number of cycles to access, type of the bus, etc.), the actual floorplan of the final chip is in the thermal model. Any of the positions suggested in Fig. 2b as "Mem i" would be valid: no matter at what side of the processor we place the memory in the FPGA, since it will be modeled as being underneath it, in a different layer, as it appears in Fig. $2 \mathrm{~b}$. 
a

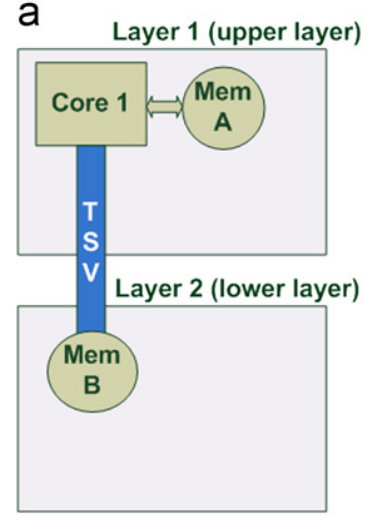

b

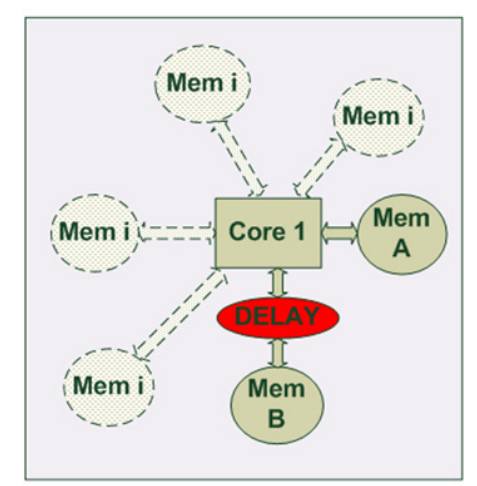

Fig. 2. (a) Modeled floorplan and (b) FPGA components mapping.

\section{Active cooling model for 2D/3D MPSoCs}

Modeling of the 3D stacked architecture with liquid cooling can be accomplished in three steps: (i) defining a grid-level thermal resistor-capacitor (RC) network of 2D/3D chip stacks, (ii) adding models for the interlayer material and TSVs distribution and (iii) modeling water flowing in independent thermal cell layers, which represent microchannels in the stacks. These three steps are detailed in this section.

\section{1. $R C$ network for $2 D / 3 D$ stacks}

2D/3D thermal modeling can be accomplished using an automated model that forms the RC circuit for certain grid dimensions. In this work, the model proposed in [15] is used, which has been extended to include 3D modeling capabilities as discussed in [17]. The extension for the existing multi-layered thermal modeling provides a new interlayer material model to include the TSVs (cf. Section 4.2) and the microchannels (cf. Section 4.3). To model the heterogeneous characteristics of the interlayer material including the TSVs and microchannels, we introduce two major differences to other works: (1) as opposed to having a uniform thermal resistivity value of the layer, our infrastructure enables having various resistivity values for each grid, (2) the resistivity value of the cell can vary at runtime.

To have a better view of what the system looks like, take a look at Fig. 3. In this figure, we can see that different tiers contain the processing cores and memories, interleaved with interlayer material, where the microchannels and TSVs are.

The interlayer material is divided into a grid, where each grid cell except for the cells of the microchannels has a fixed thermal resistance value depending on the characteristics of the interface material and TSVs. As presented in [34,35] for our considered TSV density (less than $1 \%$ of total chip area), we assume a homogeneous via distribution on the die, and calculate the combined resistivity of the interface material based on the TSV density. Then, the thermal resistivity of the microchannel cells is computed based on the liquid flow rate through the cell, and the characteristics of the liquid at runtime.

\subsection{Through-silicon-vias modeling}

In order to model the effect of TSVs on the thermal behavior of 3D MPSoCs, we performed a complete study to determine which modeling granularity is required.

As explained in [36,37], the thermal conductivity of a thermal via region is determined by its density of thermal vias. In Fig. 2 of

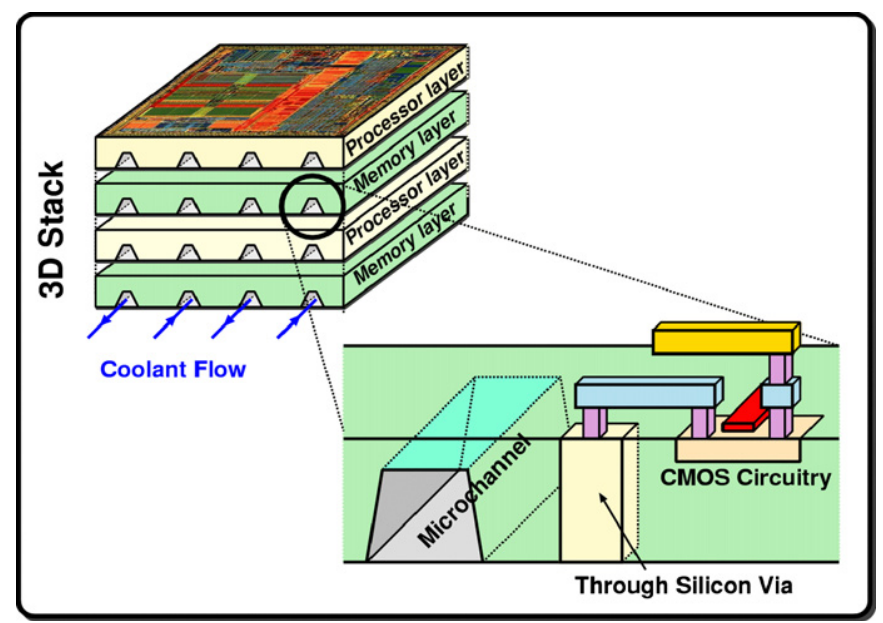

Fig. 3. Detail of the microchannels and TSVs in the 3D stack chip.

[37], we can see the resistivity as a function of vias density. As shown in [34], for small values of the TSV density (up to 1-2\%), the effect on the temperature profile is limited to only a few degrees. Therefore, through the rest of this paper, we can safely assume that the effect of the TSV insertion to the heat capacity of the interface material is negligible, since we keep the area overhead of TSVs below $1 \%$, a very small percentage of the interface material area. This TSVs thermal interference, however, can also be used as an advantage to control on-chip temperatures, through thermal via planning [35]. We assign a TSV density to each unit based on its functionality and system design choices (a crossbar structure requires a high TSV density, while a processing core does not require any modeling of TSV interference).

The TSV dimensions are set to $10 \mu \mathrm{m} \times 10 \mu \mathrm{m}$, and a minimum spacing of $10 \mu \mathrm{m}$ from each side of the TSV is employed. In fact, the experiments developed in the calibrated 3D stack thermal simulation model of the 5-tier stack (see experiments Section 5.3, Linear model vs. finite-element simulations) indicate that a blocklevel granularity provides very similar results to providing the exact locations of TSVs, while it has a very important complexity reduction in transient thermal analysis.

\subsection{Active (liquid) cooling modeling}

Active cooling properties (i.e., liquid cooling) have been modeled using additional layers of thermal cells with different cooling thermal conductance and resistance properties than silicon and metal layers, using IBM's technology $[12,13]$. In such a 3D system, the local junction temperature can be accurately computed with conjugate heat and mass transfer modeling. The complexity of the resulting model (for the fluid, only) is in the range of billion nodes to be simulated. Instead of using this expensive, in terms of computation, method, we propose (and validate) an alternative model based on resistive networks. It runs at a fraction of the computation requirements, while keeping the loss in accuracy negligible, thus, making it suitable for our realtime emulation platform.

We characterize the chip stack using a porosity model, i.e., the cavities are seen as 2D-porous media, and we study the fluidsolid thermal field-coupling. The parameters we have to calculate, then, are the equivalent thermal resistance and the permeability of the cells that form the net.

In Fig. 4a, we observe a single heat transfer unit cell of the resistor network representing the thermal field-coupling of the 2D-porous media ( $\left.T_{\text {fluid }}\right)$ with the adjacent 3D-solid walls $\left(T_{\text {wall }}\right)$. 


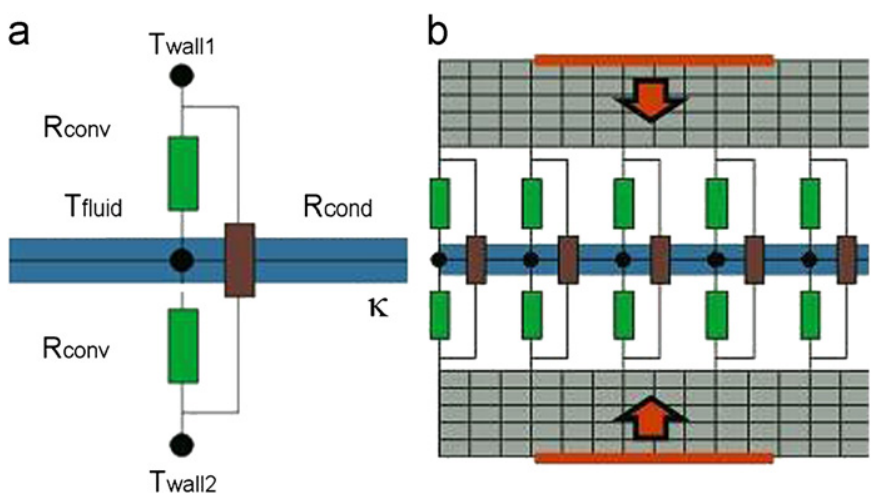

Fig. 4. (a) Heat transfer unit cell and (b) modeling the whole channel.

Here, we represent field-coupling to transfer heat from solid to fluid (as green resistors) and solid to solid (as brown resistors), the convective thermal resistance $\left(R_{\text {conv }}\right)$ and the cavity permeability $(\kappa)$.

The permeability is defined through Darcy's law as

$\nabla p=-\frac{\mu}{\kappa} \vec{v}_{\text {Darcy }}$

with the linear dependence of pressure gradient $(\nabla p)$ on the superficial velocity, also called the Darcy velocity $\left(v_{\text {Darcy }}\right)$, and the dynamic viscosity $(\mu)$ as material coefficient. The Darcy velocity is the average fluid velocity $\left(v_{\text {bulk }}\right)$ in the cavity multiplied with the cavity porosity $(\varepsilon)$ :

$\vec{v}_{\text {Darcy }}=\varepsilon \vec{v}_{\text {bulk }}$

The cavity porosity is the ratio of the cavity fluid volume $\left(V_{\text {fluid }}\right)$ to the total cavity volume including the fluid and solid part $\left(V_{t o t}\right)$ :

$\varepsilon=\frac{V_{\text {fluid }}}{V_{\text {tot }}}$

The projected convective thermal resistance $\left(R_{\text {conv }}\right)$ mapping the heat transfer on a single cavity side is computed by

$R_{\text {conv }}=\frac{\bar{T}_{\text {wall }}-\bar{T}_{\text {fluid }}}{\dot{q}_{1}}$

with average wall $\left(T_{\text {wall }}\right)$ temperature and fluid $\left(T_{\text {fluid }}\right)$ temperature, and the heat flux $\left(q_{1}\right)$ dissipated on one cavity wall in case of a symmetric heat flux boundary conditions.

The solid-solid (tier to tier) conductive thermal resistance is defined as

$R_{\text {cond }}=\frac{t_{\text {cavity }}}{k_{\text {solid }}(1-\varepsilon)}$

with cavity thickness $\left(t_{\text {cavity }}\right)$, pin or channel wall thermal conductivity $\left(k_{\text {solid }}\right)$ and porosity $(\varepsilon)$. The permeability and convective thermal resistance of a microchannel at fully developed boundary layers is independent of the Reynolds number and fluid velocity, respectively.

The values used to model the microchannel cells are depicted in Table 1. As shown in Fig. 4b, the whole channel is modeled by replicating this discrete element. As fluid advances through the channel, it removes the excess of heat from the adjacent walls.

\section{Experimental setup and results}

In this section, we first explain through different experiments how the proposed 3D thermal model was validated, including the liquid fluid modeling aspect. Then, to conclude, we present a
Table 1

Effective model parameters for interconnect pitch of $100 \mu \mathrm{m}$.

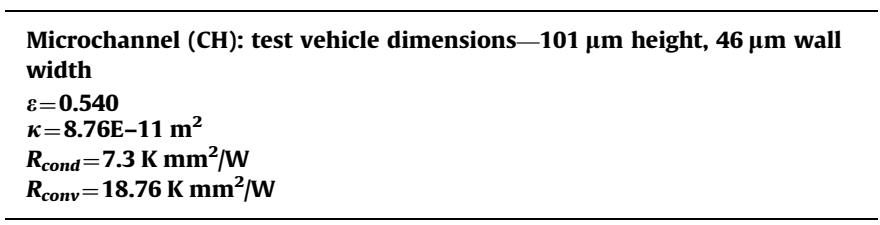

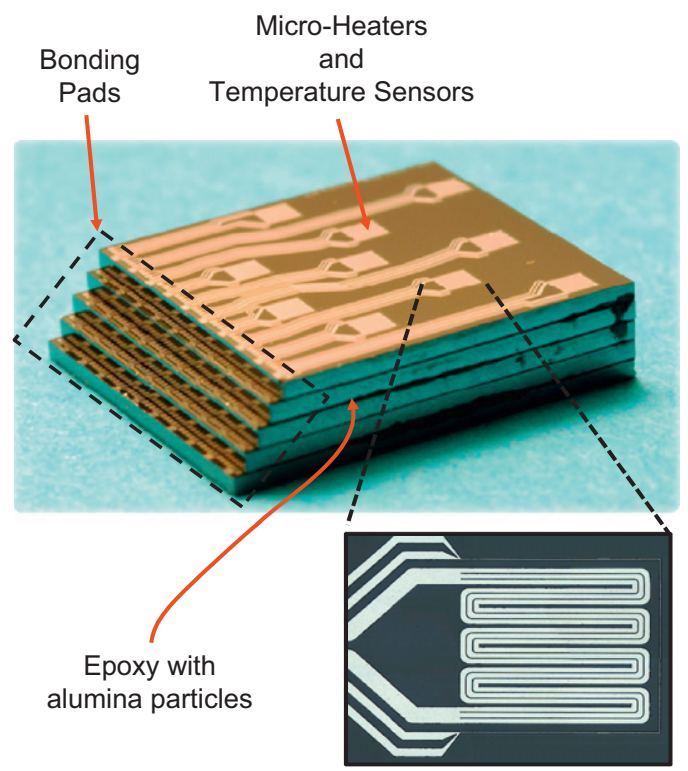

Fig. 5. Manufactured 5-tier chip stack for 3D thermal library validation.

complete use case that illustrates how designers can use the proposed emulation framework to speed-up their design phase of 3D MPSoCs.

\section{1. $3 D$ thermal model validation}

The initial 2D RC thermal model was validated using experimental data from industrial partners ([14,15,37]) for the manufacturing technologies of 2D MPSoCs. In order to calibrate the 3D version of the thermal library, we manufactured a 5-tier 3D chip stack (see Fig. 5), and carried out exhaustive experiments on it to characterize the possible inaccuracy with respect to our model, and tune it accordingly.

The structure of each of the layers of our stacked chip can be observed in Fig. 6. The "Di" elements are heat sources, modeling cores in a typical 3D MPSoC (10 microheaters of $1 \mathrm{~mm}^{2}$ per layer). Surrounding each core, there is a thermal sensor to monitor the temperature inside the stack and check the heat dissipated and the heat interactions between neighbouring cores. These sensors (i.e., thin filaments around the heaters) are resistance temperature detectors: the temperature of the heater creates a variation in the resistance of the sensor. Then, the temperature can be obtained by injecting a test (fixed) current, observing the voltage drop at both extremities of the sensor and applying the resistivity temperature dependence of the metal (platinum).

In the process to validate the 3D thermal model, we first verified the correct behavior of the heaters and sensors. Next, we studied the lateral (intra-layer) and vertical (inter-layer) heat propagation. In all cases, the figures show comparative results between the measurements in the different sensors and the simulation. In the following figures, D stands for device (see Fig. 6), 
$\mathrm{H}$ stands for heater ( $\mathrm{Hi}$ is the heater around $\mathrm{Di}$ ), and $\mathrm{L}$ stands for layer.

\subsubsection{Heaters-sensors validation}

In order to verify the correct thermal behavior of the heaters and sensors, we inject different currents and read back the temperature measured in the corresponding sensors. In the following figures, we indicate the consumed amount of Watts, which is derived by the measured currents and the resistances of the heaters of the 3D stack case study. First, in Fig. 7 we show the thermal validation results of device D1 $(\mathrm{S}+\mathrm{H}$ : Heater and sensor are at the same location) for the different layers. The $Y$ axis shows the change in temperature with respect to ambient temperature (set to $293 \mathrm{~K}$. ).

We repeated the same experiments for different devices in the 3D-IC stack and Table 2 outlines the error of the model. As this table indicates for D1 and D2, the modeling error is very small

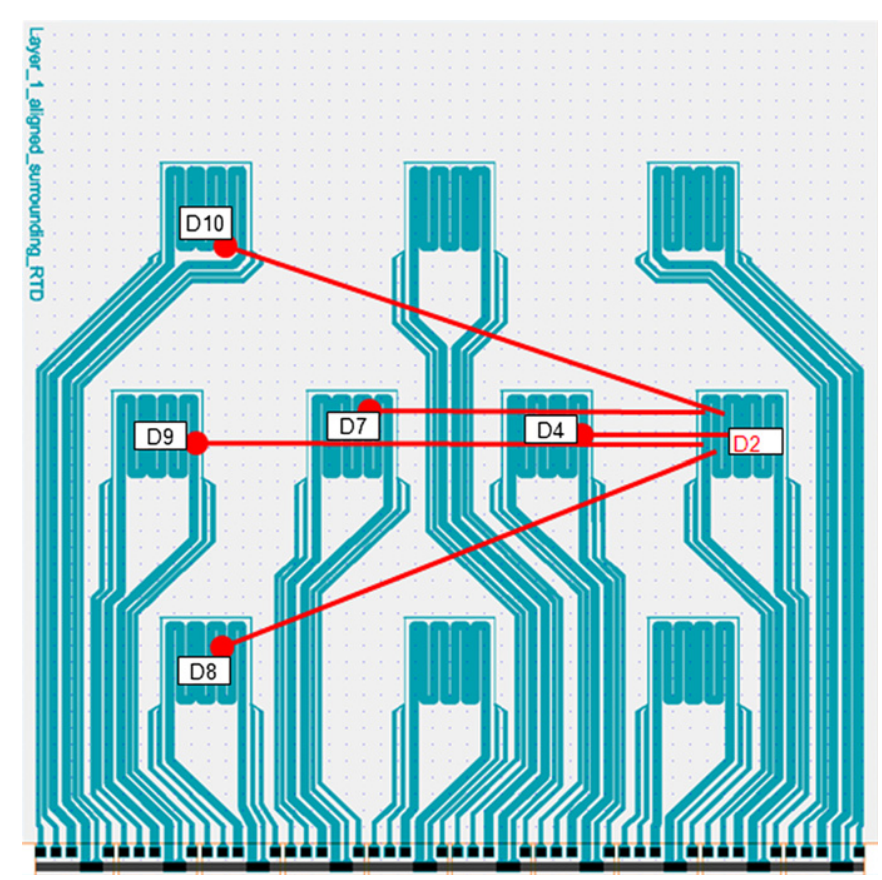

Fig. 6. One layer of the 3D stack chip: microheaters and sensors.

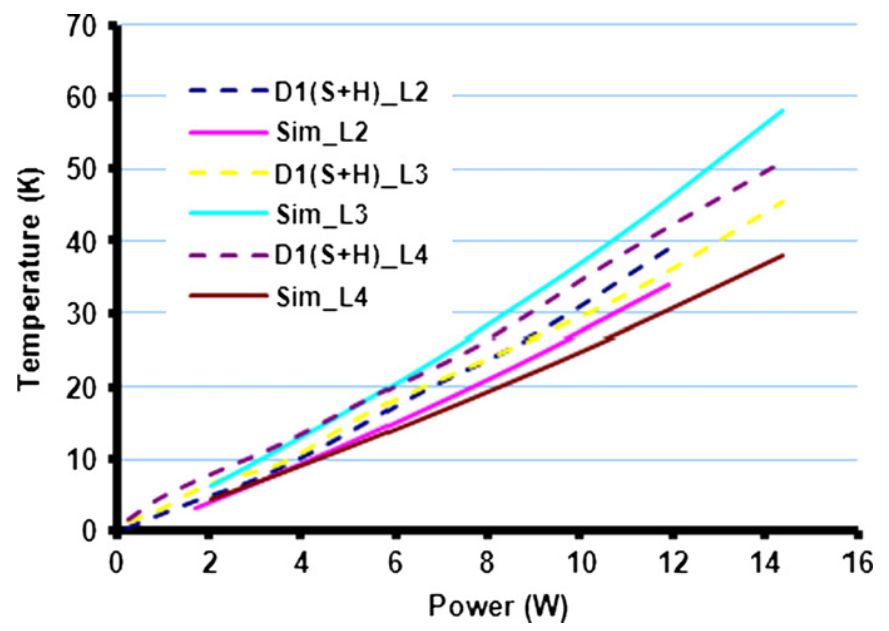

Fig. 7. Validation of thermal model in component D1, indicating measured temperature change with respect to the ambient (both in simulation and in 3D chip measurements).
Table 2

Summary of thermal model validation for different heaters and sensors.

\begin{tabular}{lllll}
\hline \multicolumn{5}{l}{ Simulation results vs measurements } \\
\hline Power (W) & D1L2 (\%Error) & D1L4 (\%Error) & D2L2 (\%Error) & D2L4 (\%Error) \\
\hline 3.0 & 0.2 & 1.3 & 0.5 & 0.7 \\
4.7 & 0.4 & 1.9 & 0.5 & 1.2 \\
6.7 & 0.8 & 2.2 & 0.5 & 1.6 \\
9.1 & 0.9 & 3.1 & 0.8 & 2.0 \\
11.9 & 1.5 & 3.7 & 0.6 & 2.4 \\
\hline
\end{tabular}

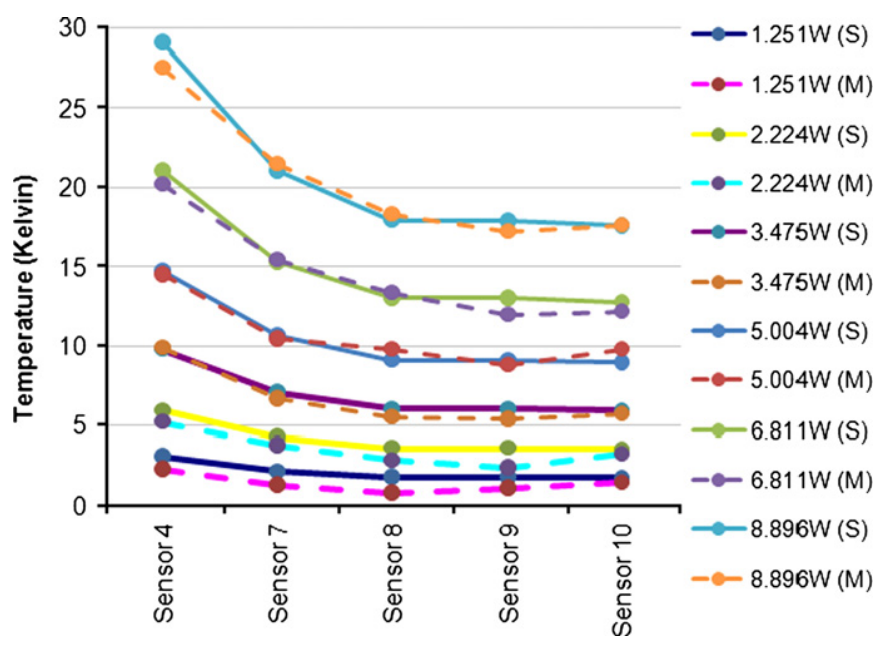

Fig. 8. Lateral heat transfer measurement indicating measured temperature change with respect to the ambient.

( $2.4 \%$, as worst case, at $11.9 \mathrm{~W}$ power value), and lower layers show slightly better accuracy. Indeed, with higher layers, it is more difficult to estimate the values, since the convection effect makes the temperature depend on the underlying layers.

\subsubsection{Intra-layer validation}

In order to characterize the lateral heat diffusion in a given layer of the 3D stack, one heater (in the example, see Fig. 6, D02 in Layer 2: D2L2) is excited with different current levels and, for each level, measurements are made at the different sensors within the same die. Thus, results in Fig. 8 provide information on the behavior of the lateral temperature distribution as function of the distance of the sensor from the heat source within a layer, using both measured values and simulated ones for different power figures. Thus, the first two values in the legend show the case when $1.251 \mathrm{~W}$ are burned in the heater; the first one (solid line) is the value obtained in the thermal model simulator ( $S$ ), while the second one (dashed line) is the measurement from the chip (M).

\subsubsection{Inter-layer validation}

The heater D2L2 is again excited with different current levels. In this case, for each current level, temperature measurements are made at the sensors in devices D02 of Die 2, Die 3, Die 4 and Die 5. In Fig. 9, then, we observe the behavior of the temperature distribution as function of the vertical distance of the sensor from the heat source in different layers of the 3D stack. The graph compares again simulation results (solid line) versus measurements (dashed line) for different power figures.

Overall, the percentage of average errors between the simulated and measured thermal resistance using the proposed $3 \mathrm{D}$ RC-based thermal model stays under $1 \%$ for all the 
sensor-heater configurations in the intra-layer case, and less than $3 \%$ in the inter-layer case.

In the next section, we focus on the modeling of the liquid cooling channels, and compare our efficient fluid modeling method against the classical finite-element simulation.

\subsection{Linear RC model vs. finite-element simulations}

In this experiment it has been evaluated the accuracy of the proposed RC-based thermal model with respect to the finiteelement-based transient thermal analysis of 3D liquid coolingbased MPSoCs. To this end, it has been compared the temperature evolution at the junction (cf. Eq. (2)) using finite-element simulations [13] (red solid line) with the estimated temperature of the linear model we developed (yellow dashed line).

The results are shown in Fig. 10, which indicate that the variations between both types of simulations are less than $1.5 \%$ on average (encircled area). Furthermore, while the proposed emulation framework using the simple liquid cooling model for straight channels can calculate the junction temperature evolution in the order of few milliseconds, the detailed finite-element simulation can take few hours. Thus, it illustrates the potential of linear thermal estimation methods for simple geometries of liquid microchannels using a laminar flow regime.

\subsection{Real-life framework test case}

The proposed HW/SW thermal emulation framework for 2D/3D MPSoCs has been compared with different SW thermal libraries for

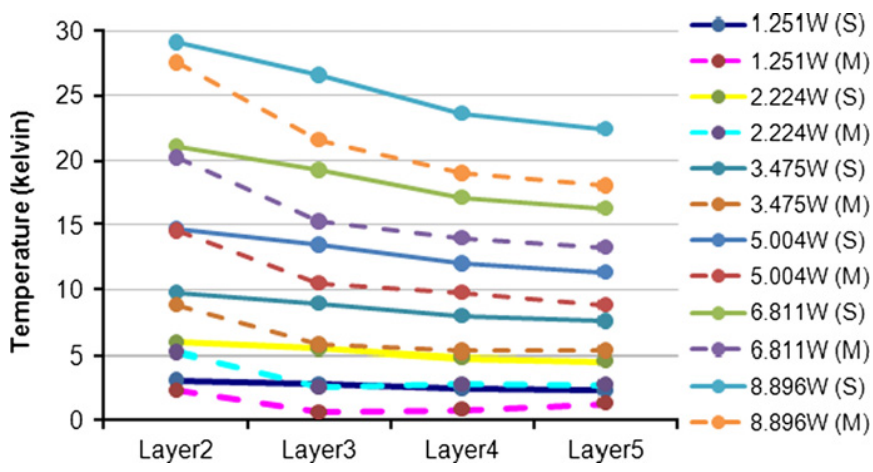

Fig. 9. Vertical heat transfer measurement indicating measured temperature change with respect to the ambient.

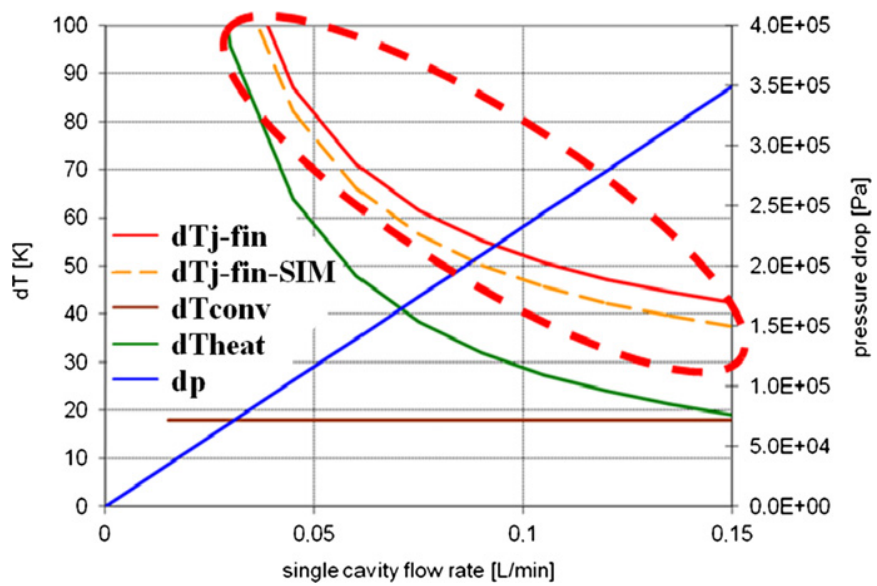

Fig. 10. Temperature evolution at the junction in liquid-cooling based systems using the finite-element simulation and the proposed model for straight liquid cooling channels.

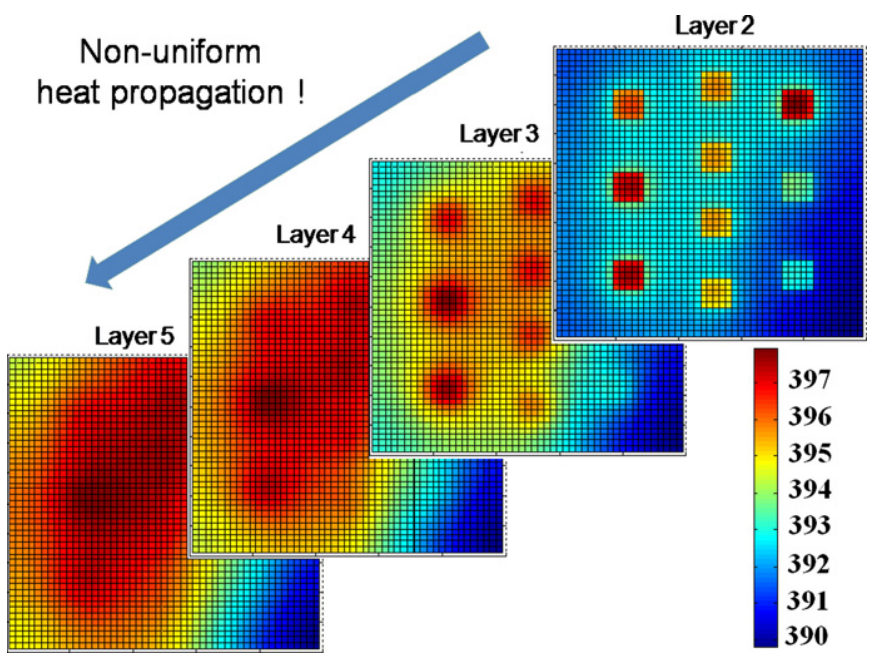

Fig. 11. Transient thermal map of the 3D MPSoC emulated system.

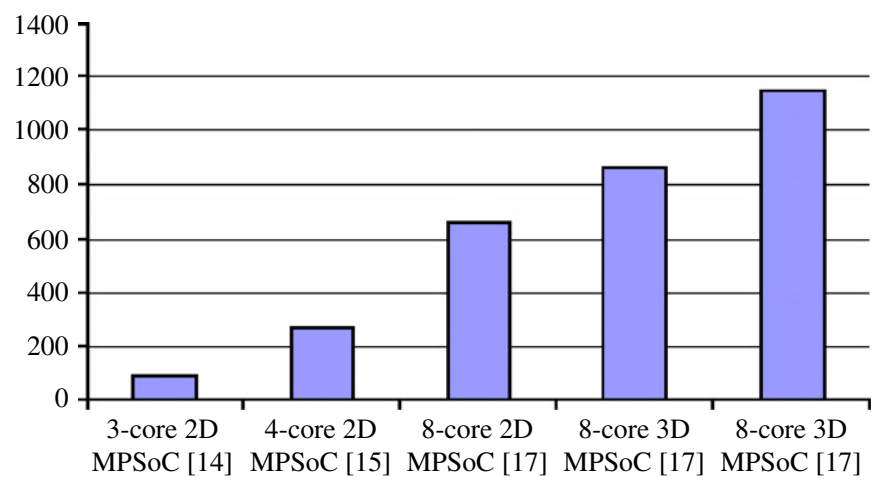

Fig. 12. Simulation speed-ups of the proposed HW-SW thermal emulation framework for transient thermal analysis with respect to state-of-the-art 2D/3D thermal simulators.

2D/3D MPSoCs [6,7,17], while running intensive SW processing kernels.

Detailed temperature maps of the modeled system can be extracted at any particular transient moment of the emulation of a certain 3D MPSoC (see Fig. 11). The obtained results are depicted in Fig. 12 and show significant speed-ups with respect to state-ofthe-art temperature estimation frameworks [14-17]. In particular, these results outline that the proposed modeling approach scales significantly better than state-of-the-art SW simulators for the transient thermal analysis. In fact, the results of the exploration of 2D thermal behavior on a commercial 8-core MPSoC [2] have shown that the proposed thermal emulation can achieve speedups of more than $800 \times$ with respect to thermal simulators.

Moreover, the thermal exploration of 3D MPSoCs with active cooling (liquid) modeling shows even larger speed-ups (more than $1000 \times$ ) due to power extraction and thermal synchronization overhead in thermal simulators $[6,7,17]$.

\section{Conclusions}

2D and emerging 3D MPSoC architectures have been proposed as a promising solution to exploit the available area in forthcoming computing systems. In this paper, we have presented a new HW/SW FPGA-based emulation framework that enables the rapid analysis of run-time thermal behavior in 2D/3D MPSoCs with active liquid cooling. The experimental results have shown that the proposed framework obtains detailed transient thermal 
exploration with a speed-up of more than $1000 \times$ with respect to cycle-accurate MPSoC simulators, even more when active (liquid) cooling effects are considered in the overall thermal system analysis. Furthermore, almost no loss in thermal estimation accuracy (less than $3 \%$ ) is experienced with respect to classical (and very time-consuming) finite-element simulations. Overall, this HW/SW thermal emulation approach is a promising mechanism to perform long-time transient behavior characterization in 2D and 3D MPSoC stacks. We have presented a real-life case study where the proposed framework can be efficiently used to evaluate the performance of liquid cooling in a 5-tier 3D chip.

\section{Acknowledgments}

This research has been partially funded by the Nano-Tera.ch RTD Project CMOSAIC (Ref. 123618), which is financed by the Swiss Confederation and scientifically evaluated by SNSF. This work has also been supported by the Spanish Government Grant TIN2008-00508 and the MEC Consolider Ingenio CSD00C-0720811 Grant of the Spanish Council of Science and Innovation.

\section{References}

[1] D. Pham, et al., Design and implementation of a first-generation cell processor, Proc. ISSCC (2005).

[2] P. Kongetira, et al., Niagara: a 32-way multithreaded SPARC processor, IEEE Micro (2005)

[3] Tilera Corporation, Tilera's 64-core architecture, 2008, 〈www.tilera.com/ products/processors.php $>$.

[4] W. Davis, et al., Demystifying 3D ICs: the pros and cons of going vertical, IEEE Des. Test Comput. (2005).

[5] M. Healy, et al., Multiobjective microarchitectural floorplanning for 2D and 3D ICs, IEEE Trans.CAD (2007)

[6] K. Skadron, et al., Temperature-aware microarchitecture: modeling and implementation (hot-spot simulator), IEEE TACO (2004).

[7] G. Paci, et al., Exploring temperature-aware design in low-power MPSoCs, Proc. Des. Autom. Test Eur. (2006).

[8] M.-N. Sabry, High-precision thermal models, IEEE TCPT (2005)

[9] Cadence Palladium II, 2005, 〈http://www.cadence.com $\rangle$.

[10] ARM integrator AP, 2004, 〈http://www.arm.com $\rangle$

[11] Emulation Engineering, Zebu models, 〈http://www.eve-team.com $\rangle$.
[12] T. Brunschwiler, et al., Direct liquid-jet impingement cooling with micronsized nozzle array and distributed return architecture, Proc. ITHERM (2006).

[13] T. Brunschwiler, et al., Interlayer cooling potential in vertically integrated packages, Microsyst. Technol. (2008).

[14] F. Mulas, et al., Thermal balancing policy for streaming computing on multiprocessor architectures, Proc. Des. Autom. Test Eur. (2008).

[15] D. Atienza, et al., A fast HW/SW FPGA-based thermal emulation framework for multi-processor system-on-chip, Proc. DAC (2006).

[16] B. Richard, et al., Numerical Analysis, Brooks Cole, 2000

[17] A.K. Coskun, et al., Dynamic thermal management in 3D multicore architectures, Proc. Des. Autom. Test Eur. (2009).

[18] A. Jerraya, et al., in: Multiprocessor SoCs, Morgan Kaufmann, 2005.

[19] G. Braun, et al., Processor/memory co-exploration on multiple abstraction levels, Proc. Des. Autom. Test Eur. (2003).

[20] P.S. Magnusson, A Simics, et al., Full system simulation platform, IEEE Comput. (2002).

[21] P. Paulin, A Stepnp, et al., System-level exploration platform for network processors, IEEE Des. Test Comput. (2002).

[22] Coware, Convergence and Lisatek product lines, 2006, 〈http://www.coware. com $>$.

[23] ARM, PrimeXSys Platform Architecture and Methodologies, White Paper, 2005, 〈http://www.arm.com/ >.

[24] Heron Engineering, SoCemulation, 2004, 〈http://www.hunteng.co.uk $\rangle$.

[25] M.D. Nava, et al., An open platform for developing MPSoC, IEEE Comput. (2005).

[26] Y. Nakamura, A fast HW/SW co-verification method for SoC by using a C/CH simulator and FPGA emulator with shared register communication, Proc. DAC (2004).

[27] Mentor Graphics, Platform express and primecell, 2005, 〈http://www. mentor.com/>.

[28] Synopsys, Realview Max-sim ESL Exploration Framework, 2004, 〈http:// www.synopsys.com/ $>$.

[29] T.-Y. Chiang, et al., Thermal analysis of heterogeneous 3-D ICs with various integration scenarios, Proc. IEDM (2001)

[30] A. Rahman, et al., Thermal analysis of three-dimensional integrated circuits, Proc. IITC (2001).

[31] P. Leduca, et al., Challenges for 3d IC integration: bonding quality and thermal management, Proc. IITC (2007).

[32] K. Puttaswamy, et al., Thermal analysis of a 3d die-stacked high-performance microprocessor, Proc. GLSVLSI (2006).

[33] A. Jain, et al., Thermal modeling and design of 3D integrated circuits, Proc. ICTTPES (2008)

[34] C. Zhu, Z. Gu, L. Shang, R.P. Dick, R. Joseph, Three-dimensional chipmultiprocessor run-time thermal management, IEEE Trans. CAD (2008).

[35] J. Cong, et al., Thermal via planning for 3-D ICs, Proc. ICCAD (2005) 745-752.

[36] B. Goplen, et al., Placement of thermal vias in 3-d ICs using various thermal objectives, IEEE Trans. CAD (2006).

[37] K. Ayse, Coskun et al., Dynamic thermal management in 3d multicore architectures, Proc. Des. Test. Eur. (2009). 